\title{
Pairing of charged particles in a quantum plasmoid
}

\author{
Maxim Dvornikov \\ Institute of Physics, University of São Paulo, \\ CP 66318, CEP 05315-970 São Paulo, SP, Brazil and \\ Pushkov Institute of Terrestrial Magnetism, Ionosphere \\ and Radiowave Propagation (IZMIRAN), \\ 142190 Troitsk, Moscow Region, Russia \\ E-mail: maxim.dvornikov@usp.br
}

\begin{abstract}
We study a quantum spherically symmetric object which is based on radial plasma oscillations. Such a plasmoid is supposed to exist in a dense plasma containing electrons, ions, and neutral particles. The method of creation and annihilation operators is applied to quantize the motion of charged particles in a self-consistent potential. We also study the effective interaction between oscillating particles owing to the exchange of a virtual acoustic wave, which is excited in the neutral component of plasma. It is shown that this interaction can be attractive and result in the formation of ion pairs. We discuss possible applications of this phenomenon in astrophysical and terrestrial plasmas.
\end{abstract}

\section{Introduction}

The studies of quantum effects in the dynamics of charged particles in dense plasmas is a rapidly developing branch of the modern plasma physics [1]. Numerous effects such as the behavior of quantum dots [2], the exciton dynamics [3] etc, unusual for classical systems, were recently reported to exist when quantum dynamics is taken into account. Note that quantum effects in astrophysical plasmas are also important in the evolution of compact stars (see, e.g., Ref. [4). However, as it was mentioned in Ref. [5], still there is a lack of understanding how to correctly account for the quantum dynamics in plasma physics. In particular, a careful anal- ysis of applicability of any of the approaches for the description of quantum plasmas should be made.

In the present work we shall discuss the quantum dynamics of a certain class of plasma objects. We shall study a spherically symmetric plasmoid based on radial oscillations of charged particles. Note that such a configuration of plasma oscillations was previously analyzed in connection to the studies of the Langmuir waves collapse [6]. We also mention that spherically symmetric plasma oscillations can be a theoretical model of stable natural plasmoids [7, 8, 9, 10, 11].

We start in Sec. 2 with the formulation of a model for a quantum spherical plasmoid based 
on radial plasma oscillations. Then, in Sec. 3 the motion of charged particles is quantized using the general ideas of Sec. 2. In Sec. 4 we carry out a quantization of acoustic waves which are excited in the neutral component of plasma existing in our system. We describe the effective interaction between charged particles owing to the exchange of an acoustic wave and analyze when this interaction can be attractive in Sec. 5. In Sec. 6] we study the possibility of the formation of bound states of oscillating charged particles in a spherical plasmoid. Finally, in Sec. 7 some applications are considered. In Sec. 8 we briefly summarize our results.

\section{Model of a quantum plas- moid}

One of the main approaches for the quantitative studies of quantum effects in dense plasmas consists in accounting for the quantum pressure term in the Euler equation, i.e., for instance, the pressure becomes [12],

$$
p \rightarrow p-\frac{\hbar^{2}}{2 m^{2} \sqrt{n}} \nabla^{2} \sqrt{n},
$$

where $m$ is the particle mass and $n$ is the number density. Note that the modification (1) is likely to account for small corrections to the dynamics of classical plasmas. For example, there is an additional term in the dispersion relation for Langmuir plasma waves, $\omega^{2}=\omega_{\mathrm{cl}}^{2}(\mathbf{q})+\hbar^{2} \mathbf{q}^{4} / 4 m_{e}^{2}$ (see, e.g., Ref. [13]), where $\omega$ is the frequency of plasma oscillations, $\omega_{\mathrm{cl}}(\mathbf{q})$ is the classical dispersion relation, $m_{e}$ is the electron mass, and $\mathbf{q}$ is the wave vector. Thus this approach for the description of the plasma dynamics seems to be the quasi-classical method for the studies of quantum effects in plasma physics. Therefore, to describe the dynamics of a strongly correlated system of charged particles, this method is unlikely to be valid and the new approach has to be developed.

If we are interested in the studies of a quantum object based on oscillations of charged particles in plasma [9, the main problem in this description is the construction of a ground state of this many body system. The ground state based on the particles wave functions taken in the form of plane waves, as it is typically made in the condensed matter physics, is inappropriate since it does not fully reflect the dynamical features of the system. Thus we should build the ground state using the wave functions corresponding to the oscillatory motion of charged particles.

The dynamics of plasma oscillations on the classical level was studied in Ref. 14] using the Lagrange variables method. In this formalism, the time evolution of a particular charged particle is analyzed. It was found that the separation of positive and negative charges in plasma results in the appearance of oscillations of lightest charged particles, i.e. electrons, with the Langmuir frequency $\omega_{e}=\sqrt{4 \pi e^{2} n_{e}^{(0)} / m_{e}}$, where $e>0$ is the proton charge and $n_{e}^{(0)}$ is the equilibrium number density of electrons. It means that the equation of motion of an electron has the form,

$$
\ddot{\mathbf{r}}=-\omega_{e}^{2} \mathbf{r} .
$$

Thus one can suggest that the perturbed motion of a single electron in plasma is governed by the quadratic potential $V(\mathbf{r})=m_{e} \omega_{e}^{2} r^{2} / 2$. In this case one can reproduce the classical equation of motion (2).

We should also mention that the idealized picture described above when only electrons participate in plasma oscillations is never implemented. In a realistic case ions are also in- 
volved in the plasma motion. We can consider ion-acoustic waves as an example of ion oscillations in plasma. The dispersion relation for these waves reads [15],

$$
\omega(\mathbf{q})=\omega_{i} \frac{\lambda_{\mathrm{D}}|\mathbf{q}|}{\sqrt{1+\lambda_{\mathrm{D}}^{2}|\mathbf{q}|^{2}}}
$$

where $\omega_{i}=\omega_{e} \sqrt{m_{e} / m_{i}}$ is the Langmuir frequency for ions, $\lambda_{\mathrm{D}}=\sqrt{k_{\mathrm{B}} T_{e} / 4 \pi e^{2} n_{e}^{(0)}}$ is the Debye length, $T_{e}$ is the electron temperature, and $k_{\mathrm{B}}$ is the Boltzmann constant. Considering rather short waves with $|\mathbf{q}| \gg 1 / \lambda_{\mathrm{D}}$ we get that $\omega \approx \omega_{i}$. Note that the damping of such waves is small [15].

The oscillatory motion of ions can be also excited when various electron-ion and electronelectron nonlinearities are taken into account (see, e.g., Refs. [6, 16, 17]). It makes Eq. (2) highly nonlinear. As a rule, these nonlinearities reduce the frequency of electron oscillations. In this case we can assume that electrons still perform oscillatory motion but with another frequency $\omega_{0}<\omega_{e}$. Note that, in some cases, quantum effects can be more strongly marked for ions rather than for electrons [18].

Thus we will take that the motion of a charged particle in plasma is governed by the potential $V(\mathbf{r})=m \omega_{0}^{2} r^{2} / 2$, where $m$ stays for the electron or ion mass and $\omega_{0}$ is the oscillations frequency of charged particles. As we mentioned above, oscillations of ions should be treated as the collective effect.

Let us assume that the dynamics of charged particles in a quantum plasmoid is also determined by the same potential $V(\mathbf{r})$. Using this assumption, the construction of the ground state of this plasma structure will be made in Sec. 3, Of course, to have a correlated many particles system, the typical size of such an object should be quite small. We shall obtain some estimates of the length scale of these plasmoids in Sec 7. Note that, in Ref. [14], the cases of planar, cylindrical, and spherical plasma oscillations were studied. In our work we will be mainly concentrated on the description of spherically symmetric quantum plasmoids.

\section{Quantization of the charged particles motion}

To describe the quantum dynamics of a particle in plasma we choose the Schrödinger picture, i.e. the operators of observables will be time independent. If we study the stationary states of particles, with the total wave function $\sim \exp \left(-\mathrm{i} \frac{E t}{\hbar}\right) \psi$, where $E$ is the particle energy, then the Schrödinger equation for the coordinate wave function $\psi$ has the form,

$$
E \psi=\hat{H}_{\mathrm{fq}} \psi, \quad \hat{H}_{\mathrm{fq}}=-\frac{\hbar^{2}}{2 m} \nabla^{2}+\frac{m \omega_{0}^{2} r^{2}}{2},
$$

where we take into account that particles in a plasmoid move in the potential $V(\mathbf{r})$, defined in Sec. 2,

We shall be interested in the spherically symmetric solutions of Eq. (4). Using the results of Ref. [19] we can express the properly normalized total wave function in the following form:

$$
\begin{aligned}
\psi_{n \sigma}(r) & =\frac{1}{\sqrt{4 \pi(2 n+1) !}} \frac{(-1)^{n}}{2^{n}} \\
& \times\left(\frac{m^{3} \omega_{0}^{3}}{\pi \hbar^{3}}\right)^{1 / 4} \exp \left[-\frac{m \omega_{0}}{2 \hbar} r^{2}\right] \\
& \times \frac{H_{2 n+1}\left(\sqrt{\frac{m \omega_{0}}{\hbar}} r\right)}{\sqrt{\frac{m \omega_{0}}{\hbar}} r} \chi_{\sigma},
\end{aligned}
$$

where $H_{n}(z)$ is the Hermite polynomial, $\chi_{\sigma}$ is the spin wave function, and $\sigma= \pm 1$ is the spin 
variable. Quantum states, described by the wave $E_{0}$, functions (5), have the energies,

$$
E_{n}=\hbar \omega_{0}\left(2 n+\frac{3}{2}\right)
$$

where $n=0,1,2, \ldots$ is the radial quantum number.

To secondly quantize the system, we introduce the operator valued wave functions,

$$
\hat{\psi}(r)=\sum_{n \sigma} \hat{a}_{n \sigma} \psi_{n \sigma}(r)
$$

where $\hat{a}_{n \sigma}$ is the annihilation operator. One can also write down the analogous expression for the Hermitian conjugate wave function which will contain the creation operator $\hat{a}_{n \sigma}^{\dagger}$. We shall suppose that ions are half integer spin particles, i.e. they are fermions like electrons. Thus, the operators $\hat{a}_{n \sigma}$ and $\hat{a}_{n \sigma}^{\dagger}$ satisfy the canonical anticommutation relation $\left[\hat{a}_{n \sigma}, \hat{a}_{n^{\prime} \sigma^{\prime}}^{\dagger}\right]_{+}=\delta_{n n^{\prime}} \delta_{\sigma \sigma^{\prime}}$ for both electrons and ions.

Using Eqs. (4) and (5) we can express the total energy of the system of charged particles as

$$
\begin{gathered}
E_{\mathrm{cp}}=\int \mathrm{d}^{3} \mathbf{r} \hat{\psi}^{\dagger}(r) \hat{H}_{\mathrm{fq}} \hat{\psi}(r) \\
=\sum_{n \sigma} E_{n} \hat{a}_{n \sigma}^{\dagger} \hat{a}_{n \sigma} .
\end{gathered}
$$

Finally we replace the total energy

$$
E_{\mathrm{cp}} \rightarrow \hat{H}_{\mathrm{cp}}=\sum_{n \sigma} E_{n} \hat{a}_{n \sigma}^{\dagger} \hat{a}_{n \sigma}
$$

with the secondly quantized Hamiltonian.

Now we can calculate the energy, $E_{0}$, of the ground state of the system. If the total number of particles, $N$, is even, using Eq. (9) we get for

$$
\begin{aligned}
E_{0}= & 2 \sum_{n=0}^{n_{\mathrm{F}}} \hbar \omega_{0}\left(2 n+\frac{3}{2}\right) \\
& =2 \hbar \omega_{0}\left(n_{\mathrm{F}}^{2}+\frac{5}{2} n_{\mathrm{F}}+\frac{3}{2}\right) .
\end{aligned}
$$

If $N$ is odd, we analogously obtain that

$$
\begin{gathered}
E_{0}=2 \sum_{n=0}^{n_{\mathrm{F}}-1} \hbar \omega_{0}\left(2 n+\frac{3}{2}\right)+\hbar \omega_{0}\left(2 n_{\mathrm{F}}+\frac{3}{2}\right) \\
=2 \hbar \omega_{0}\left(n_{\mathrm{F}}^{2}+\frac{3}{2} n_{\mathrm{F}}+\frac{3}{4}\right)
\end{gathered}
$$

Note that, if $N \rightarrow \infty$, both Eqs. (10) and (11) approach the same limit $E_{0} \rightarrow 2 \hbar \omega_{0} n_{\mathrm{F}}^{2}$. Here $n_{\mathrm{F}}$ stays for the maximal number of an occupied energy level. We can call it the Fermi number, in the analogy to the Fermi momentum.

Let us define the effective size of a plasmoid, $R$, as the position of the last maximum of the function $\left|\psi_{n_{\mathrm{F}}}(r)\right|^{2}$. If $n_{\mathrm{F}}$ is great, this $\max -$ imum is approximately achieved at the classical turn point 20 , i.e. when $\hbar \omega_{0}\left(2 n_{\mathrm{F}}+3 / 2\right)=$ $m \omega_{0}^{2} R^{2} / 2$. Accounting for this fact, we get that

$$
n_{\mathrm{F}} \approx \frac{m \omega_{0} R^{2}}{4 \hbar}
$$

Using Eq. (12) we can express the ground state energy in terms of the plasmoid radius as $E_{0} \approx$ $m^{2} \omega_{0}^{3} R^{4} / 8 \hbar$.

Note that our plasmoid model is based on the linear approximation of noninteracting particles. It corresponds to the Hartree-Fock approximation. As we mentioned in Sec. 2, if we account for various (classical or quantum) electron-ion and electron-electron interactions, it will lead to the nonlinear terms in the equation of motion (2) of a particle and impose a certain length scale in 
the system (see, e.g., Refs. [16, 17]). One the examples of such interaction is later discussed in Sec. 5.

The ground state of a spherical plasmoid was constructed under the assumption of the negligible particle temperature. The temperature corrections will wash out the lowest energy levels with $E_{n} \lesssim k_{\mathrm{B}} T$, where $T$ is the electron or ion temperature. Taking into account the fact that particles possessing the lowest energy levels are concentrated near the center of a plasmoid, it means that plasma oscillations are the most intensive in a spherical shell. The internal radius of this shell is determined by the thermal effects and the external one by the nonlinear interactions between particles. Such kind of the spherical plasmoid structure is in agreement with the numerical simulations made in Ref. [17.

\section{Quantization of the acoustic field}

As we mentioned in Sec. 3, the temperature in plasma should be not quite high for the existence of a spherical quantum plasma structure. It means that, along with the charged particles, there should be a quite significant fraction of neutral atoms or molecules in plasma. Plasma oscillations will inevitably result in collisions with neutral atoms leading to the generation of acoustic waves. In this Section we shall quantize acoustic waves inside a spherical plasmoid.

The hydrodynamics equations, which govern the acoustic waves propagation, have the form,

$$
\begin{aligned}
\frac{\partial n_{n}}{\partial t}+\nabla\left(n_{n} \mathbf{v}_{n}\right) & =0 \\
\frac{\partial \mathbf{v}_{n}}{\partial t}+\left(\mathbf{v}_{n} \nabla\right) \mathbf{v}_{n}+\frac{1}{n_{n} m_{n}} \nabla p_{n} & =0
\end{aligned}
$$

where $n_{n}$ is the number density of neutral particles, $\mathbf{v}_{n}$ is the neutral gas velocity, $p_{n}\left(n_{n}\right)$ is the pressure of the gas, and $m_{n}$ is the neutral particle mass.

Let us expand the parameters $n_{n}, \mathbf{v}_{n}$, and $p_{n}$ as

$$
\begin{aligned}
& n_{n}=n_{n}^{(0)}+n_{1}+\cdots, \\
& \mathbf{v}_{n}=\mathbf{v}_{1}+\cdots, \\
& p_{n}=p_{n}^{(0)}+\left(\frac{\partial p_{n}}{\partial n_{n}}\right)_{0}\left(n_{n}-n_{n}^{(0)}\right)+\cdots,
\end{aligned}
$$

where $n_{n}^{(0)}$ and $p_{n}^{(0)}$ are the equilibrium values of the number density and the pressure as well as $n_{1}$ and $\mathbf{v}_{1}$ are the small perturbations of the number density and the velocity. Using Eqs. (13) and (14) we get two independent wave equations for $n_{1}$ and $\mathbf{v}_{1}$,

$$
\begin{aligned}
\frac{\partial^{2} \mathbf{v}_{1}}{\partial t^{2}}-c_{\mathrm{s}}^{2} \nabla\left(\nabla \cdot \mathbf{v}_{1}\right) & =0 \\
\frac{\partial^{2} n_{1}}{\partial t^{2}}-c_{\mathrm{s}}^{2} \nabla^{2} n_{1} & =0
\end{aligned}
$$

where

$$
c_{s}=\frac{1}{m_{n}^{1 / 2}} \sqrt{\left(\frac{\partial p_{n}}{\partial n_{n}}\right)_{0}},
$$

is the sound velocity. Although we linearize Eq. (14), the influence of charged particles on the neutral component of plasma can be accounted for by renormalizing the sound velocity (17), which is regarded as a phenomenological parameter.

The spherically symmetric solution of Eq. (16) has the form,

$$
n_{1}(\mathbf{r}, t)=e^{-\mathrm{i} \omega_{k} t} f_{k}(r), \quad f_{k}(r)=\frac{\sin k r}{k r}
$$


where $\omega_{k}=c_{s} k$. Note that the coordinate function obeys the following identities:

$$
\begin{aligned}
& \int \frac{\mathrm{d}^{3} \mathbf{r}}{(2 \pi)^{3}} f_{k}(r) f_{p}(r)=\delta^{3}(\mathbf{k}-\mathbf{p}), \\
& \int \frac{\mathrm{d}^{3} \mathbf{k}}{(2 \pi)^{3}} f_{k}(r) f_{k}\left(r^{\prime}\right)=\delta^{3}\left(\mathbf{r}-\mathbf{r}^{\prime}\right),
\end{aligned}
$$

where delta-functions should be understood as $\delta^{3}\left(\mathbf{k}-\mathbf{k}^{\prime}\right)=\delta\left(k-k^{\prime}\right) / 4 \pi k^{2}$.

Following Ref. [21], we introduce the potential of the velocity as $\mathbf{v}_{1}=\nabla \varphi_{1}$. Using Eqs. (15)(18) we obtain the general expressions for $n_{1}$ and $\varphi_{1}$ as

$$
\begin{aligned}
n_{1}(r, t)= & \int \frac{\mathrm{d}^{3} \mathbf{k}}{(2 \pi)^{3 / 2}}\left(\frac{\hbar n_{n}^{(0)}}{2 m_{n} \omega_{k}}\right)^{1 / 2} \\
& \times k f_{k}\left(b_{k} e^{-\mathrm{i} \omega_{k} t}+b_{k}^{\dagger} e^{\mathrm{i} \omega_{k} t}\right), \\
\varphi_{1}(r, t)= & \int \frac{\mathrm{d}^{3} \mathbf{k}}{(2 \pi)^{3 / 2}}\left(\frac{\hbar}{2 m_{n} n_{n}^{(0)} \omega_{k}}\right)^{1 / 2} \\
& \times c_{s} f_{k}\left(-\mathrm{i} b_{k} e^{-\mathrm{i} \omega_{k} t}+\mathrm{i} b_{k}^{\dagger} e^{\mathrm{i} \omega_{k} t}\right),
\end{aligned}
$$

where $b_{k}$ and $b_{k}^{\dagger}$ are the Fourier coefficients.

The total energy of the acoustic field has the form,

$$
E_{\mathrm{ph}}=\frac{1}{2} \int \mathrm{d}^{3} \mathbf{r} m_{n}\left[n_{n}^{(0)} \nabla \varphi_{1}^{2}+\frac{c_{s}^{2}}{n_{n}^{(0)}} n_{1}^{2}\right] .
$$

Now, if we replace $b_{k}$ and $b_{k}^{\dagger}$ in Eq. (20) by the annihilation and the creation operators, $\hat{b}_{k}$ and $\hat{b}_{k}^{\dagger}$, we can express $E_{\mathrm{ph}}$, which should be regarded as the secondly quantized Hamiltonian of the phonon field, in the following way:

$$
\begin{aligned}
E_{\mathrm{ph}} \rightarrow \hat{H}_{\mathrm{ph}}= & \int \mathrm{d}^{3} \mathbf{k} \hbar \omega_{k} \hat{b}_{k}^{\dagger} \hat{b}_{k} \\
& + \text { divergent terms. }
\end{aligned}
$$

To derive Eq. (22) we suggest that the operators $\hat{b}_{k}$ and $\hat{b}_{k}^{\dagger}$ obey the canonical commutation relation for bosonic operators, $\left[\hat{b}_{k}, \hat{b}_{k^{\prime}}^{\dagger}\right]_{-}=\delta^{3}\left(\mathbf{k}-\mathbf{k}^{\prime}\right)$. Note the "divergent terms" in Eq. (22), containing $\delta(0)$, can be removed by the normal ordering of operators.

Note that, in this Section, we used the Heisenberg picture where operators turn out to be time dependent, cf. Eq. (20). Using results of Ref. 22] one can express these operators in the Schrödinger picture, which will be used in Sec. 5 . Such a transformation is equivalent to setting $t=0$ in Eq. (20).

\section{Interaction between charged particles and acoustic waves}

We have already mentioned in Sec. 4 that charged particles can interact with neutral atoms, which are present in the system. Thus charged particles should also interact with acoustic waves, which are generated by spherically symmetric plasma oscillations. In this Section we derive the secondly quantized Hamiltonian which describes this interaction. Then we exclude the acoustic part of the total Hamiltonian and reduce it to the nonlinear charged particles Hamiltonian.

We suggest that charged particles scatter off the density perturbations of neutral atoms or molecules. Thus the energy of the interaction of a charged particle with an acoustic field has the form [23],

$$
V(\mathbf{r})=\int \mathrm{d}^{3} \mathbf{r}^{\prime} K\left(\mathbf{r}-\mathbf{r}^{\prime}\right) n_{1}\left(\mathbf{r}^{\prime}\right),
$$

where $K\left(\mathbf{r}-\mathbf{r}^{\prime}\right)$ is the energy of the interaction between a charged particle placed at $\mathbf{r}$ and a neutral particle which is at $\mathbf{r}^{\prime}$. Using Eq. (23) we 
can derive the secondly quantized Hamiltonian of the interaction between charged particles and acoustic waves as

$$
\begin{aligned}
\hat{H}_{\mathrm{cp}-\mathrm{ph}}= & \int \mathrm{d}^{3} \mathbf{r} \hat{\psi}^{\dagger}(\mathbf{r}) V(\mathbf{r}) \hat{\psi}(\mathbf{r}) \\
& =K_{0} \int \mathrm{d}^{3} \mathbf{r} \hat{\psi}^{\dagger}(\mathbf{r}) \hat{\psi}(\mathbf{r}) \hat{n}_{1}(\mathbf{r}),
\end{aligned}
$$

where $\hat{\psi}$ and $\hat{n}_{1}$ are given in Eqs. (7) and (20) respectively. To derive Eq. (24) we suggest that $K\left(\mathbf{r}-\mathbf{r}^{\prime}\right)=K_{0} \delta^{3}\left(\mathbf{r}-\mathbf{r}^{\prime}\right)$. This approximation corresponds to a contact interaction between charged and neutral particles.

Using Eqs. (7) and (20) we can cast $\hat{H}_{\mathrm{el}-\mathrm{ph}}$ to the form,

$$
\begin{aligned}
\hat{H}_{\mathrm{cp}-\mathrm{ph}}= & \int \frac{\mathrm{d}^{3} \mathbf{k}}{(2 \pi)^{3 / 2}} \sum_{n s \sigma} D_{n s}(k) \\
& \times \hat{a}_{n \sigma}^{\dagger} \hat{a}_{s \sigma}\left(\hat{b}_{k}+\hat{b}_{k}^{\dagger}\right),
\end{aligned}
$$

where

$$
\begin{aligned}
D_{n s}(k)= & K_{0} k\left(\frac{\hbar n_{n}^{(0)}}{2 m_{n} \omega_{k}}\right)^{1 / 2} \\
& \times \int \mathrm{d}^{3} \mathbf{r} \psi_{n}(r) \psi_{s}(r) f_{k}(r),
\end{aligned}
$$

is the matrix element. With help of Eqs. (5) and (18) we can compute $D_{n s}(k)$ at big $n$ and $s$ in the explicit form,

$$
\begin{aligned}
D_{n s}(k) \approx & \frac{K_{0}}{4(n s)^{1 / 4}}\left(\frac{n_{n}^{(0)} m \omega_{0}}{2 m_{n} \omega_{k}}\right)^{1 / 2} \\
& \times[\operatorname{sign}(2 \sqrt{n}-2 \sqrt{s}+\xi) \\
& +\operatorname{sign}(2 \sqrt{n}+2 \sqrt{s}-\xi) \\
& -\operatorname{sign}(2 \sqrt{n}-2 \sqrt{s}-\xi)-1],
\end{aligned}
$$

where $\xi=k \sqrt{\hbar / m \omega_{0}}$.
To exclude the acoustic degrees of freedom we make the canonical transformation of the Hamiltonian in Eq. (25),

$$
\begin{aligned}
\hat{H}_{\mathrm{cp}-\mathrm{ph}} & \rightarrow e^{-\hat{S}} \hat{H}_{\mathrm{cp}-\mathrm{ph}} e^{\hat{S}}, \\
\hat{S}= & \int \frac{\mathrm{d}^{3} \mathbf{k}}{(2 \pi)^{3 / 2}} \sum_{n s \sigma} D_{n s}(k) \hat{a}_{n \sigma}^{\dagger} \hat{a}_{s \sigma} \\
& \times\left(\frac{\hat{b}_{k}}{E_{s}-E_{n}-\hbar \omega_{k}}\right. \\
& \left.+\frac{\hat{b}_{k}^{\dagger}}{E_{s}-E_{n}+\hbar \omega_{k}}\right) .
\end{aligned}
$$

It can be seen that, after this transformation, the total Hamiltonian, $\hat{H}=\hat{H}_{0}+\hat{H}_{\mathrm{cp}-\mathrm{ph}}$, transforms into $\hat{H} \rightarrow \hat{H}_{0}+\frac{1}{2}\left[\hat{H}_{\mathrm{cp}-\mathrm{ph}} ; \hat{S}\right]$, where $\hat{H}_{0}=$ $\hat{H}_{\mathrm{cp}}+\hat{H}_{\mathrm{ph}}$. Averaging over the acoustic ground state and assuming that there are no external phonons, we get the total Hamiltonian in the form,

$$
\begin{aligned}
\hat{H}= & \sum_{n \sigma} E_{n} \hat{a}_{n \sigma}^{\dagger} \hat{a}_{n \sigma} \\
& +\int \frac{\mathrm{d}^{3} \mathbf{k}}{(2 \pi)^{3}} \sum_{n n^{\prime} s s^{\prime} \sigma \sigma^{\prime}} D_{n s}(k) D_{n^{\prime} s^{\prime}}(k) \\
& \times \hat{a}_{n \sigma}^{\dagger} \hat{a}_{n^{\prime} \sigma^{\prime}}^{\dagger} \hat{a}_{s^{\prime} \sigma^{\prime}} \hat{a}_{s \sigma} \\
& \times \frac{\hbar \omega_{k}}{\left(E_{s}-E_{n}\right)^{2}-\left(\hbar \omega_{k}\right)^{2}} .
\end{aligned}
$$

Note that the Hamiltonian (29) contains both the free charged particles term and the interaction between charged particles owing to the virtual acoustic wave exchange. Let us examine when this effective interaction can be attractive. We remind that charged particles in a plasmoid oscillate with a rather high frequency $\sim \omega_{0}$. In collisions with neutral atoms these charged particles will generate acoustic waves with the typical frequency $\sim \omega_{0}[24]$. It means that the phonon 
energy in Eq. (29) is $\hbar \omega_{k} \sim \hbar \omega_{0}$. Using Eq. (6) we get that $E_{n} \sim \hbar \omega_{0} n$. It means that the effective interaction can be attractive only if $E_{s}=E_{n}$ or $s=n$. Thus there is an attraction between a pair of charged particles which are at the same energy level.

\section{Bound states of charged par- ticles}

In the previous Section we have found that the exchange of a virtual acoustic wave can result in the attractive interaction between oscillating charged particles in plasma. In this Section we study this process in details and show that under certain conditions charged particles can form pairs.

Before we proceed it should be noted that charged particles with parallel spins cannot occupy the same energy level, because of the Pauli principle. We remind that ions in our system are supposed to be fermions. Thus we should exclude the contribution of charged particles with parallel spins from the effective interaction (29). It mean that the summation in the nonlinear term should be made over the opposite spin indexes $\sigma^{\prime}=-\sigma$.

We should also mention that up to now we studied the case of constant number of particles. To avoid this restriction, we use the standard technique of shifting the energy levels $E_{n} \rightarrow$ $e_{n}=E_{n}-\mu$, where $\mu$ is the chemical potential of the system. Now the number of particles can change, but we should calculate the chemical potential.

Finally, taking into account all these comments we can transform the Hamiltonian (29) to the following form:

$$
\begin{aligned}
\hat{H}= & \sum_{n \sigma} e_{n} \hat{a}_{n \sigma}^{\dagger} \hat{a}_{n \sigma} \\
& -\sum_{n n^{\prime} \sigma} F_{n n^{\prime}} \hat{a}_{n \sigma}^{\dagger} \hat{a}_{n^{\prime},-\sigma}^{\dagger} \hat{a}_{n^{\prime},-\sigma} \hat{a}_{n \sigma},
\end{aligned}
$$

where the new matrix element can be explicitly calculated on the basis of Eq. (27) as

$$
\begin{aligned}
F_{n n^{\prime}} & =V_{0} \frac{\sqrt{n}+\sqrt{n^{\prime}}-\left|\sqrt{n}-\sqrt{n^{\prime}}\right|}{\sqrt{n n^{\prime}}}, \\
V_{0} & =\frac{K_{0}^{2} n_{n}^{(0)}}{8 \pi^{2} m_{n} c_{s}^{2}}\left(\frac{m \omega_{0}}{\hbar}\right)^{3 / 2} .
\end{aligned}
$$

It is worth mentioning that $F_{n n^{\prime}}>0$ for any $n$ and $n^{\prime}$. It means that the effective interaction described by the Hamiltonian (30) is really attractive.

To diagonalize the Hamiltonian (30) we introduce the new operators $\hat{A}_{0 n}$ and $\hat{A}_{1 n}$ by means of the Bogolyubov transformation,

$$
\begin{aligned}
& \hat{a}_{n+}=u_{n} \hat{A}_{0 n}+v_{n} \hat{A}_{1 n}^{\dagger}, \\
& \hat{a}_{n-}=u_{n} \hat{A}_{1 n}-v_{n} \hat{A}_{0 n}^{\dagger},
\end{aligned}
$$

where the real coefficients $u_{k}$ and $v_{k}$ satisfy the relation $u_{n}^{2}+v_{n}^{2}=1$. Note that the new operators obey the canonical anticommutation relations for fermion operators. Substituting these operators in Eq. (30) we get the Hamiltonian in the form,

$$
\hat{H}=E_{0}^{\prime}+\hat{H}_{2}+\hat{H}_{2}^{\prime}+\ldots,
$$

where

$$
E_{0}^{\prime}=2 \sum_{n} v_{n}^{2}\left[e_{n}-F_{n n} u_{n}^{2}\right]
$$

is the ground energy,

$$
\begin{aligned}
\hat{H}_{2}= & \sum_{n}\left[e_{n}\left(u_{n}^{2}-v_{n}^{2}\right)+4 F_{n n} u_{n}^{2} v_{n}^{2}\right] \\
& \times\left(\hat{A}_{1 n}^{\dagger} \hat{A}_{1 n}+\hat{A}_{0 n}^{\dagger} \hat{A}_{0 n}\right)
\end{aligned}
$$


is the energy of the new quasiparticles, and

$$
\begin{aligned}
\hat{H}_{2}^{\prime}= & 2 \sum_{n} u_{n} v_{n}\left[e_{n}-F_{n n}\left(u_{n}^{2}-v_{n}^{2}\right)\right] \\
& \times\left(\hat{A}_{1 n} \hat{A}_{0 n}+\hat{A}_{0 n}^{\dagger} \hat{A}_{1 n}^{\dagger}\right),
\end{aligned}
$$

is the nondiagonal part of the quasiparticles interaction. Note that in Eq. (33) we omit terms higher than quadratic ones.

The nondiagonal interaction (36) can be vanishing in two cases. Firstly, when either $u_{n}=1$ and $v_{n}=0$ or $u_{n}=0$ and $v_{n}=1$. It corresponds to the trivial solution, which is equivalent to the transition to the particles-holes representation. Note that the ground state energy (34) is not negative for this trivial solution: $E_{0}^{\prime} \geq 0$.

The second situation is implemented if the attraction between charged particles is rather strong, i.e. when

$$
F_{n n}>\left|e_{n}\right| .
$$

In this case we can choose the coefficients $u_{k}$ and $v_{k}$ as

$$
\begin{aligned}
u_{n}^{2} & =\frac{1}{2}\left(1+\frac{e_{n}}{F_{n n}}\right), \\
v_{n}^{2} & =\frac{1}{2}\left(1-\frac{e_{n}}{F_{n n}}\right) .
\end{aligned}
$$

The ground state energy (34) now becomes negative,

$$
E_{0}=-\sum_{n} \frac{\left(F_{n n}-e_{n}\right)^{2}}{2 F_{n n}}<0 .
$$

It means that the state of the system, corresponding to the new quasiparticles, is more favorable energetically than in the trivial case discussed above. The diagonal part of the Hamiltonian (35) has the form,

$$
\hat{H}_{2}=\sum_{n} F_{n n}\left(\hat{A}_{1 n}^{\dagger} \hat{A}_{1 n}+\hat{A}_{0 n}^{\dagger} \hat{A}_{0 n}\right) .
$$

Using Eqs.(31) and (40) one can find the energy of quasiparticles $E_{n}^{\prime}=2 V_{0} / \sqrt{n}$.

The chemical potential of the system can be calculated using the expression $N=\left\langle\Phi_{0}^{\prime}|\hat{N}| \Phi_{0}^{\prime}\right\rangle$, where $\left|\Phi_{0}^{\prime}\right\rangle$ is the wave function of the ground state satisfying the conditions

$$
\hat{A}_{0 n}\left|\Phi_{0}^{\prime}\right\rangle=0, \quad \text { and } \quad \hat{A}_{1 n}\left|\Phi_{0}^{\prime}\right\rangle=0,
$$

and

$$
\hat{N}=\sum_{n \sigma} \hat{a}_{n \sigma}^{\dagger} \hat{a}_{n \sigma},
$$

is the number of particles operator.

Using Eqs. (32), (38), (41), and (42) we can express the number of particles as

$$
N=2 \sum_{n} v_{n}^{2}=\sum_{n<n_{\mathrm{F}}^{\prime}}\left(1-\frac{e_{n}}{F_{n n}}\right),
$$

where the bound of the new state in the energy space, $n_{\mathrm{F}}^{\prime}$, is defined by Eq. (37). The quantity $n_{\mathrm{F}}^{\prime}$ is analogous to the Fermi number introduced in Sec. 3. Basing on Eqs. (6) and (43) we get the system of equations,

$$
\begin{aligned}
& N=\frac{n_{\mathrm{F}}^{\prime}}{3}+\frac{4}{15} n_{\mathrm{F}}^{\prime 5 / 2} \frac{\hbar \omega_{0}}{V_{0}}, \\
& \mu=\hbar \omega_{0}\left(2 n_{\mathrm{F}}^{\prime}+\frac{3}{2}\right)-\frac{2 V_{0}}{\sqrt{n_{\mathrm{F}}^{\prime}}},
\end{aligned}
$$

which define the quantities $n_{\mathrm{F}}^{\prime}$ and $\mu$ as functions of $N$. To derive Eq. (44) we use the approximate identity,

$$
\sum_{n<n_{\mathrm{F}}^{\prime}} n^{\alpha} \approx \int_{0}^{n_{\mathrm{F}}^{\prime}} n^{\alpha} \mathrm{d} n=\frac{\left(n_{\mathrm{F}}^{\prime}\right)^{\alpha+1}}{\alpha+1},
$$

which is valid at big $n_{\mathrm{F}}^{\prime}$.

The analysis of this Section shows that the exchange of a virtual acoustic wave between oscillating charged particles in a quantum spherical plasmoid results in the formation of pairs of 
these particles. There is a pairing of particles with oppositely directed spins. These particles should be at the same energy level. Note that this new state of plasma has less energy of the ground state compared to the situation of unpaired particles. This process is analogous to the formation of Cooper pairs of electrons in a metal. We remind that, if the temperature of a metal is significantly low, two electrons can form a bound state owing to the exchange of a virtual phonon, which is a quanta of the vibration of a crystal lattice of a metal. This process underlies the phenomenon of superconductivity.

Note that besides the oppositely directed spins, two electrons in a metal should have opposite momenta 25 . In our case the situation is analogous. Indeed, at big radial quantum numbers, $n \gg 1$, the asymptotic expansion of the charged particles wave function (5) can be expressed as

$$
\begin{aligned}
\psi_{n}(r) \sim & \frac{1}{r} \sin \left(2 \sqrt{\frac{m \omega_{0} n}{\hbar}} r\right) \\
& =\frac{1}{2 \mathrm{i} r}\left[\exp \left(2 \mathrm{i} \sqrt{\frac{m \omega_{0} n}{\hbar}} r\right)\right. \\
& \left.-\exp \left(-2 \mathrm{i} \sqrt{\frac{m \omega_{0} n}{\hbar}} r\right)\right] .
\end{aligned}
$$

Eq. (46) means that any steady state of a charged particle in a spherical plasmoid is a superposition of converging and divergent spherical running waves. The pairing of two charged particles with opposite spins happens between the states corresponding to the running waves with opposite momenta.

Let us demonstrate by means of the explicit calculation that the formation of a bound state of two charged particles is possible in our system. Suppose that there is the state of two charged particles having opposite spins,

$$
|\Psi\rangle=\sum_{n} c_{n} \hat{a}_{n,+}^{\dagger} \hat{a}_{n,-}^{\dagger}\left|\Phi_{0}\right\rangle,
$$

where $c_{n}$ are the expansion coefficients and $\left|\Phi_{0}\right\rangle$ is the ground state corresponding to the filled lowest energy states: $\hat{a}_{n \sigma}\left|\Phi_{0}\right\rangle=0$, cf. Sec. 3 Using the Hamiltonian (30) we can show that the energy corresponding to the state (47) is

$$
E_{\Psi}=\langle\Psi|\hat{H}| \Psi\rangle=2 \sum_{n}\left(E_{n}-F_{n n}\right)\left|c_{n}\right|^{2}
$$

where $E_{n}$ is given by Eq. (6). Thus the energy of the state (47) is less than the sum of energies of two non-interacting charged particles, with $F_{n n}$ being the binding energy.

Analyzing Eq. (48) it is interesting to mention that, in contrast to the formation of Cooper pairs in metal, where bound states are formed near the Fermi sphere surface, in our case the pairing of particles happens at all the energy levels. This fact can be explained by the very convenient choice of the ground state wave functions (5), which maximally account for the dynamical features of the system (see also Sec. 2). Of course, we should remind that our analysis is valid for relatively big radial quantum numbers $n$.

\section{Possible applications}

To analyze the possibility of the pairing of charged particles in a spherically symmetric plasma structure we can use Eq. (48) which gives one the energy of the bound state of two particles with opposite spins. Note that this expression is valid just before the pairing since it is based on the ground state $\left|\Phi_{0}\right\rangle$ rather than on $\left|\Phi_{0}^{\prime}\right\rangle$, defined by Eq. (41). The pairing may happen if 
$E_{n}<F_{n n}$. Using Eqs. (6) and (31), one obtains that this constraint is equivalent to

$$
n<\left(\frac{V_{0}}{\hbar \omega_{0}}\right)^{2 / 3} \text {. }
$$

Supposing that the Fermi number of a plasmoid before the pairing, given in Eq. (12), is smaller than the constraint (49), we get the following upper bound:

$$
R<R_{\mathrm{cr}}=\left(\frac{K_{0}^{2} n_{n}^{(0)}}{\pi^{2} \hbar \omega_{0} m_{n} c_{s}^{2}}\right)^{1 / 3},
$$

on the radius of a plasma structure.

Note the constraint (50) is very conservative. It means that charged particles on all energy levels can form bound states simultaneously. On the contrary, we may require that only particles at lower levels form pairs and then this process spreads to higher energy states. Thus in a realistic case the upper bound of plasmoid radius (50) may be significantly changed towards its enhancement. However the analysis of the dynamics of this phase transition requires a separate special study.

To evaluate the plasmoid radius one should define $K_{0}$, which does not depend on the macroscopic plasma characteristics. We remind that we approximated the potential $K(\mathbf{r})$ by a deltafunction. For this kind of potential, using the Born approximation, one can calculate the total cross section of the charged particles scattering off the neutral particles as $\sigma_{s}=m^{2} K_{0}^{2} / \pi \hbar^{4}$. Note that in our approximation the cross section does not depend on the particles energy, which may not be the case for some realistic potentials.

Let us first examine the possibility of pairing of charged particles inside a spherical plasmoid in a very dense medium corresponding to the inner crust of a neutron star (NS). The number density of neutral particles, i.e. neutrons, at the bottom of the inner crust is $n_{n}^{(0)} \sim$ $10^{38} \mathrm{~cm}^{-3}[26$. We will be mainly interested in the studies of the formation of pairs of protons. The number density of protons in the NS crust strongly depends on the equation of state of the NS matter. According to Ref. [27] it can be about several per cent of the neutron density. In our estimates we take that $n_{p}^{(0)} \sim 10^{36} \mathrm{~cm}^{-3}$. Thus we get that at such a density the Langmuir frequency for protons is $\omega_{0} \sim 10^{21} \mathrm{~s}^{-1}$. The sound velocity also depends on the equation of state of the nuclear matter. Nevertheless we may take it as $c_{s} \sim 10^{9} \mathrm{~cm} \cdot \mathrm{s}^{-1}$ [28], which corresponds to the chosen density of the NS crust.

Protons in the NS crust are highly degenerate. Their Fermi energy does not exceed several MeV. At such typical energies the cross section of the proton-neutron scattering is approximately constant and equals to $2 \times 10^{-23} \mathrm{~cm}^{2}[29$. This fact justifies the use of the delta-function potential in Eq. (24). Thus we can also evaluate $K_{0}$. Finally, using Eq. (50) we get that protons can form pairs in a plasmoid with $R_{\mathrm{cr}} \approx 4.6 \times 10^{-12} \mathrm{~cm}$. The obtained value for the critical radius means that there can be several hundreds of energy levels inside the plasmoid. This result is in agreement with the fact that there can be a proton superconductivity in the NS matter [30].

It should be mentioned that besides uniform dense matter, containing neutrons, protons, and electrons, nuclei can be also present in the NS crust. The presence of nuclei can affect the process of the protons pairing in frames of our model. However, at the densities corresponding to the bottom of inner crust, used in our work, the fraction of nuclei is negligible [31.

Note that the energy corresponding to the ze- 
roth level, $3 \hbar \omega_{0} / 2$, turns out to be of the order of the Fermi energy for protons. Thus thermal effects will not influence the plasmoid dynamics. We should also mention that for the pairing of protons inside the NS matter to happen, the proton-phonon interaction owing to the acoustic wave exchange should be dominant. It means that the electromagnetic interaction of protons should be screened. Supposing that electrons in the NS crust are ultrarelativistic, we get for the Debye length, $\lambda_{\mathrm{D}} \sim 10^{-13} \mathrm{~cm}$, that is much smaller than the plasmoid radius. Thus the electromagnetic interaction of protons can be omitted.

It should be noticed that the cross section of the electron-neutron scattering is several orders of magnitude smaller than that of the protonneutron scattering [32. Moreover the Langmuir frequency for electrons is much higher than that for protons. It means that the critical volume of a plasmoid becomes too small to contain enough number of particles. Thus the electron superconductivity is unlikely to exist in a spherical plasmoid inside NS.

Although one might expect that the pairing of charged particles can happen only in plasmas with very high densities, which may be encountered only in astrophysical media, we may discuss the situation when this phenomenon occurs in terrestrial conditions. Let us discuss the case when a plasmoid appears in liquid water with $n_{n}^{(0)} \sim 10^{23} \mathrm{~cm}^{-3}$. We suggest that the number density of singly ionized water ions is $n_{i}^{(0)} \sim 10^{21} \mathrm{~cm}^{-3}$. The Langmuir frequency for water ions is $\omega_{0} \sim 10^{13} \mathrm{~s}^{-1}$. The sound speed in water is $c_{s} \sim 10^{5} \mathrm{~cm} \cdot \mathrm{s}^{-1}$. Note that the total spin of a neutral water molecule is integer. Thus, if we study a singly ionized water ion, its spin should be half integer, i.e. it is a fermion.
Unfortunately, the scattering of water ions on neutral molecules of water is not very well studied. Anyway we can assume that the total cross section of such a scattering cannot be less than that for electron scattering on water molecules which can be $\sim 10^{-14} \mathrm{~cm}^{2}$ in the eV electron energy range 33 . Thus using Eq. (50) we get that $R_{\mathrm{cr}} \approx 4.6 \times 10^{-7} \mathrm{~cm}$. Again we can see that there are several hundreds of energy levels inside the plasmoid.

As in case of a plasmoid existing in the crust of NS, the pairing of electrons in a spherical plasma structure involving water ions is unlikely to happen because of the very high frequency of electron oscillations. The fact that the exchange of a virtual acoustic wave results in the cohesion of ions rather than electrons was also noticed in Ref. 23].

The typical energy of a stochastic motion of an ion is $k_{\mathrm{B}} T_{i} \sim 10^{-14} \mathrm{erg}$, where $T_{i} \approx 300 \mathrm{~K}$ is the ion temperature. Using the above estimate for the oscillations frequency, we get that the energy of the zeroth energy level is also $\sim 10^{-14} \mathrm{erg}$. Thus we can disdain the thermal effects in a spherical plasma structure with water ions. We can also check that one can neglect the electromagnetic interaction between water ions. Taking the electron temperature of $\sim 10^{3} \mathrm{~K}$ (such a temperature corresponds to $10 \%$ of the ionization potential of a hydrogen atom), we get that the Debye length is $\sim 10^{-8} \mathrm{~cm}$, which is much less than the plasmoid radius.

We have shown that the pairing of ions is possible in a plasmoid which is a radial oscillation of charged particles in plasma. It is also clear that ions play a subdominant role in plasma oscillations because of their low mobility. We may put forward a hypothesis that the pairing of electrons can also happen provided the frequency of their oscillations is significantly reduced owing 
to nonlinear electron-electron or electron-ion interactions (see, e.g., Refs. [6, 16, 17]). However, this issue requires an additional special study.

We described the formation of pairs of water ions inside a plasmoid. This process may result in the appearance of superconducting phase in plasma. Note that the idea that the plasma superconductivity may explain the stability of a natural plasma structure, called ball lightning (BL) 34], was previously discussed in Refs. [35, 36]. As we have seen above, for the pairing of charged particles to happen, the typical diameter of a plasmoid should be very small, $\sim\left(10^{-7}-10^{-6}\right) \mathrm{cm}$. The plasma structures of the similar size, based on quantum oscillations of electrons, were studied in Ref. [9]. It should be noted that previously the magnetic interaction [37] and the exchange of a virtual Langmuir wave [38] were considered as possible mechanisms which underlie the plasma superconductivity.

It should be noticed that the existence of the superconducting state of plasma inside BL can result in the presence of strong nondecaying electric currents in this kind of objects. It may explain the fact that sometimes the appearance of a natural BL magnetically affected heavy metallic objects like a church bell [39].

The results of our work can applied for the explanation of the BL creation in natural conditions. It is very difficult to excite a weakly decaying plasma oscillation. However, if we suppose that the pairing of charged particles in a quantum plasmoid, and possibly superconductivity, happens at the initial stages of plasma structure evolution, one can account for the creation of BL in a drop of rain water. Moreover our results may be used in the interpretation of the experiments with electric discharges in liquid water [40, 41, 42, where luminous spherical ob- jects, resembling a natural BL, were generated.

We should also mention that separate tiny plasmoids, each of them being a radial oscillation of charged particles, can form a composite object due to the quantum exchange interaction [43]. Note that the model of a composite BL, which is confirmed by observations 34, was also recently discussed in Ref. [44].

\section{Conclusion}

In conclusion we mention that in the present work we have developed the theory of quantum spherical plasmoids. Such a plasma structure is based on radially symmetric oscillations of charged particles in plasma. The method of linearized quantum hydrodynamics [1, 5], which is frequently used to account for quantum effects in plasmas, seems to be quasi-classical. On the contrary, our method involves quantum mechanical description of the electrons and ions evolution in a self-consistent potential which governs the oscillatory motion of charged particles. Thus our approach is valid for the description of dense quantum plasmas where quantum effects are important.

We have developed a model of quantum plasmoid which involves the secondly quantized motion of charged particles. Note that the formalism of creation and annihilation operators was also previously used in plasma physics [45]. In frames of this approach various aspects of quantum plasma dynamics, like phase transitions [46], the behavior under the influence of strong laser fields [47, the time evolution in external fields [48, and the particle trapping in a strong electromagnetic field [49, have been studied. The recent applications of the nonequilibrium Green function formalism to the solid 
state physics can be found in Ref. [50]. We also mention an alternative description of quantum plasma dynamics, which is based on Monte Carlo simulations [51]. In the present work for the first time the have used the second quantization formalism for the studies of a confined plasma stricture which has a spherical symmetry.

In Sec. 3 we have applied the general formalism to the quantization of charged particles in a spherically symmetric plasmoid. In particular we have derived the secondly quantized Hamiltonian (9) and have constructed the ground state of the system. In our work we have discussed the situation when, along with oscillating charged particles, there is a neutral component in plasma. In Sec. 4 we have secondly quantized the field of acoustic waves which are inevitably excited inside a plasmoid. The quantization of a phonon field is necessary since later we discuss possible applications which involve phonons propagation in dense media, like nuclear matter of NS and liquid water, cf. Sec. 7 .

Then, in Sec. 5, we have considered the interaction between charged particles and acoustic waves. We have derived the secondly quantized Hamiltonian of charged particles which accounts for the effective interaction owing to the exchange of virtual acoustic waves, cf. Eq. (29). We have also considered the situation when this interaction can be attractive. In Sec. 6] we have discussed the attractive interaction between charged particles in details and have shown that it can result in the transformation of the ground state of the system. We have demonstrated that charged particles, which are supposed to be $1 / 2$ spin fermions, tend to form singlet bound states which are more favorable energetically. This phenomenon is analogous to the formation of Cooper pairs in a metal, that results in the metal superconductivity.
In Sec. 7 we have discussed the possible applications of the pairing of charged particles in plasma owing to the exchange of virtual acoustic waves. Firstly, we have considered the case of a very dense plasma corresponding to the inner crust of NS. It has been shown that protons in NS matter can form pairs. This result is in agreement with the previous findings that proton superconductivity may well happen in the NS matter. Secondly, we have studied the possibility of pairing of singly ionized water ions inside a plasmoid in the terrestrial conditions. We have shown that this phenomenon can happen provided a plasma structure is created in a liquid water and the density of ions is quite high. We have also considered the implication of our results to the theoretical description of stable natural plasma objects as well as to the resent experiments where plasmoids were generated in electric discharged in water.

We have obtained that the pairing is unlikely to occur in the electron component of plasma. It happens because of the quite high frequency of electron oscillations. Thus electrons will excite acoustic waves ineffectively. Moreover we should take into account the higher electron temperature compared to that of ions. It means that the formation of bound states of electrons will be washed out by their thermal motion. This result is consistent with the claim of the authors of Ref. 23. who found that the exchange of an acoustic wave leads to the cohesion of ions rather than electrons.

\section{Acknowledgments}

I am thankful S. I. Dvornikov for helpful discussions and to FAPESP (Brazil) for a grant. 


\section{References}

[1] Manfredi G 2005 How to model quantum plasmas Topics in kinetic theory ed T Passot, C Sulem and P-L Sulem (Fields institute communications vol 46) (Providence, RI: American Math. Soc.) pp 263288 (arXiv:quant-ph/0505004)

[2] Balzer K, Bonitz M, van Leeuwen R, Stan A and Dahle N E 2009 Nonequilibrium Green's function approach to strongly correlated few-electron quantum dots Phys. Rev. B 79245306 (arXiv:0810.2425 [condmat.mes-hall])

[3] Arkhipov A S, Lozovik Yu E, Man'ko V I and Sharapov V A 2005 Center-of-mass tomography and probability representation of quantum states for tunneling Teor. Math. Phys. 142 311-323

[4] Melrose D B 2008 Quantum plasmadynamics: Unmagnetized plasmas (New York: Springer)

[5] Vladimirov S V and Tyshetskiy $\mathrm{Yu} \mathrm{O}$ 2011 On description of a collisionless quantum plasma Phys.-Usp. $54 \quad$ 1243-1256 (arXiv:1101.3856 [physics.plasm-ph])

[6] Zakharov V E 1972 Collapse of Langmuir waves Sov. Phys.-JETP 35 908-914

[7] Zheng X-H 1990 Quantitative analysis for ball lightning Phys. Lett. A 148 463-469

[8] Fedele R 1999 A possible quantum-like approach to non-conventional plasmas Proceedings of 6th Int. Symp. on Ball Lightning (Univ. of Antwerp, Antwerp, Belgium, 23-26 August 1999) ed G C Dijkhuis (Terneuzen: ZSC) pp 126-132
[9] Dvornikov M and Dvornikov S 2006 Electron gas oscillations in plasma: Theory and applications Advances in plasma physics research vol 5, ed F Gerard (New York: Nova Science Publishers) pp 197-212 (arXiv:physics/0306157)

[10] Shmatov M L 2003 New model and estimation of the danger of ball lightning $J$. Plasma Phys. 69 507-527

[11] Tennakone K 2011 Stable spherically symmetric static charge separated configurations in the atmosphere: Implications on ball lightning and earthquake lights $J$. Electrostat. $69638-640$

[12] Kuzelev M V and Rukhadze A A 1999 On the quantum mechanical description of the linear kinetics of a collisionless plasma Phys.-Usp. 42 603-605

[13] Bohm D and Pines D 1953 A collective description of electron interactions: III. Coulomb interactions in a degenerate electron gas Phys. Rev. 92 609-625

[14] Dawson J M 1959 Nonlinear electron oscillations in a cold plasma Phys. Rev. 113 383387

[15] Lifshitz E M and Pitaevskii L P 2010 Physical kinetics (Burligton: Elsevier) pp 136137

[16] Škorić M M and ter Haar D 1980 Higher electron nonlinearities in the dynamics of Langmuir collapse Physica C 98 211-221

[17] Davydova T A, Yakimenko A I and Zaliznyak Yu A 2005 Stable spatial Langmuir solitons Phys. Lett. A 336 46-52 (arXiv:physics/0408023) 
[18] Abrikosov A A 1961 Some properties of strongly compressed matter. I Sov. Phys.JETP 12 1254-1259

[19] Cohen-Tannoudji C, Diu B and Laloë F 1977 Quantum mechanics vol 1 (New York: Wiley) pp 814-823

[20] Blokhintsev D I 1964 Quantum mechanics (Dordrecht: Reidel) pp 140-141

[21] Zakharov V E and Kuznetsov E A 1997 Hamiltonian formalism for nonlinear waves Phys.-Usp. 40 1087-1116

[22] Abrikosov A A, Gor'kov L P and Dzyaloshinskii I Ye 1965 Quantum field theoretical methods in statistical physics 2nd ed (Oxford: Pergamon) pp 44-45

[23] Vlasov A A and Yakovlev M A 1978 Interaction between ions through an intermediate system (neutral gas) and problem of existence of a cluster of particles maintained by its own forces. I Theor. Math. Phys. 34124 130

[24] Dvornikov M 2012 Effective attraction between oscillating electrons in a plasmoid via acoustic wave exchange Proc. R. Soc. A 468 415-428 (arXiv:1102.0944] [physics.plasm$\mathrm{ph}]$ )

[25] Madelung O 1978 Introduction to solid-state theory (Berlin: Springer) pp 230-233

[26] Shapiro S L and Teukolsky S A 1983 Black holes, white dwarfs, and neutron stars: The physics of compact objects (New York: Wiley) p 252

[27] Shen G, Horowitz C J and Teige S 2011 New equation of state for astrophysi- cal simulations Phys. Rev. C 83035802 (arXiv:1101.3715 [astro-ph.SR])

[28] Epstein R I 1988 Acoustic properties of neutron stars Astrophys. J. 333 880-894

[29] Chadwick M B et al. 2006 ENDF/B-VII.0: Next generation evaluated nuclear data library for nuclear science and technology Nucl. Data Sheets 107 2931-3059

[30] Yakovlev D G, Levenfish K P and Shibanov Yu A 1999 Cooling neutron stars and superfluidity in their interiors Phys.Usp. 42 737-778 (arXiv:astro-ph/9906456)

[31] Haensel P, Potekhin A Y and Yakovlev D G 2007 Neutron stars I: Equation of state and structure (New York: Springer) pp 140-142

[32] Frois B and Papanicolas C N 1987 Electron scattering and nuclear structure Ann. Rev. Nucl. Part. Sci. 37 133-176

[33] Itikawa Y and Mason N 2005 Cross sections for electron collisions with water molecules J. Phys. Chem. Ref. Data 34 1-22

[34] Bychkov V L, Nikitin A I and Dijkhius G C 2010 Ball lightning investigations The atmosphere and ionosphere: Dynamics, processing and monitoring ed V L Bychkov, G V Golubkov and A I Nikitin (Dordrecht: Springer) pp 201-373

[35] Dijkhuis G C 1980 A model for ball lightning Nature 284 150-151

[36] Zelikin M I 2008 Superconductivity of plasma and fireballs J. Math. Sci. 151 3473-3496 
[37] Meierovich B E 1984 Correlation properties of charges in plasma streams and the possibility of superconductivity Phys. Scr. 29 494-499

[38] Veklenko B A 2012 Langmuir vacuum and superconductivity Plasma Phys. Rep. 38 513-519

[39] Blair A J F 1973 Magnetic fields, ball lightning and campanology Nature 243 512-513

[40] Golubnichii P I, Gromenko V M and Krutov V M 1990 Formation of long-lived glowing objects in the decay of a dense lowtemperature water plasma Sov. Phys.-Tech. Phys. 35 110-111

[41] Shabanov G D 2002 The optical properties of long-lived luminous formations Tech. Phys. Lett. 28 164-166

[42] Versteeg A, Behringer K, Fantz U, Fussmann G, Jüttner B and Noack S 2008 Longliving plasmoids from an atmospheric water discharge Plasma Sour. Sci. Technol. 17 024014

[43] Dvornikov M 2012 Quantum exchange interaction of spherically symmetric plasmoids J. Atm. Sol.-Terr. Phys. 89 62-66 (arXiv:1112.0239 [physics.plasm-ph])

[44] Meshcheryakov O 2007 Ball lightningaerosol electrochemical power source or a cloud of batteries Nanoscale Res. Lett. 2 319-330

[45] Kremp D, Schlanges M and Kraeft W-D 2005 Quantum statistics of nonideal plasmas (Berlin: Springer) pp 65-115
[46] Schlanges M, Bonitz $\mathrm{M}$ and Tschttschjan A 1995 Plasma phase transition in fluid hydrogen-helium mixtures Contrib. Plasma Phys. 35 109-125

[47] Kremp D, Bornath Th, Bonitz M and Schlanges M 1999 Quantum kinetic theory of plasmas in strong laser fields Phys. Rev. E 60, 4725-4732

[48] Bonitz M, Bornath T, Kremp D, Schlanges M and Kraeft W D 1999 Quantum kinetic theory for laser plasmas: Dynamical screening in strong fields Contrib. Plasma Phys. 39, 329-347

[49] Fromm A, Bonitz M and Dufty J 2008 Quantum kinetic theory of trapped particles in a strong electromagnetic field $A n n$. Phys. 323 3158-3174

[50] Balzer K and Bonitz M 2009 Nonequilibrium properties of strongly correlated artificial atoms-a Green's functions approach J. Phys. A: Math. Theor. 42214020 (arXiv:0810.2633 [cond-mat.mes-hall])

[51] Filinov A V, Bonitz M and Lozovik Yu E 2001 Wigner crystallization in mesoscopic 2D electron systems Phys. Rev. Lett. 86 3851-3854 